Provided for non-commercial research and education use. Not for reproduction, distribution or commercial use.

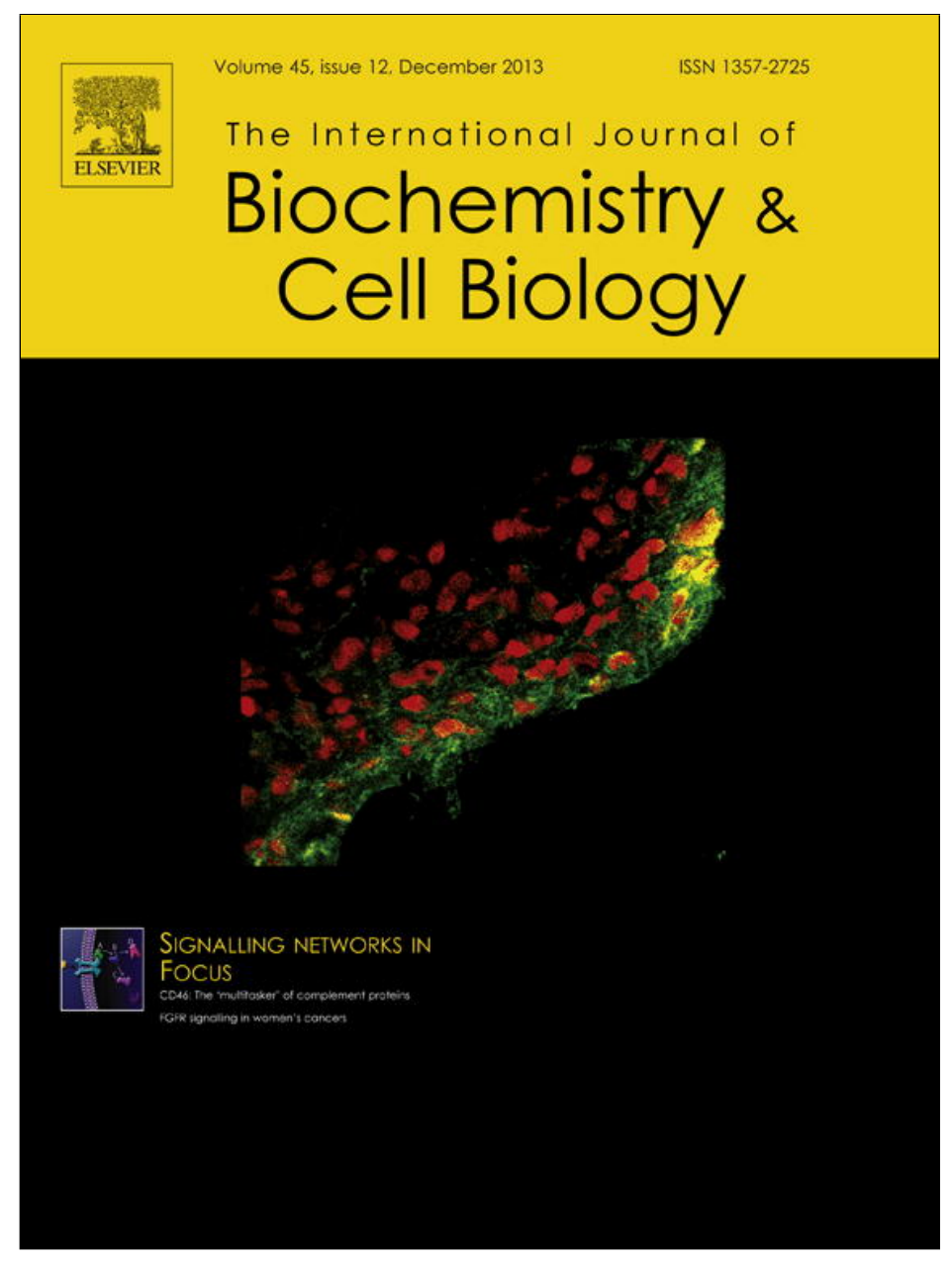

This article appeared in a journal published by Elsevier. The attached copy is furnished to the author for internal non-commercial research and education use, including for instruction at the authors institution and sharing with colleagues.

Other uses, including reproduction and distribution, or selling or licensing copies, or posting to personal, institutional or third party websites are prohibited.

In most cases authors are permitted to post their version of the article (e.g. in Word or Tex form) to their personal website or institutional repository. Authors requiring further information regarding Elsevier's archiving and manuscript policies are encouraged to visit:

http://www.elsevier.com/authorsrights 


\title{
Expression of carbohydrate-antigen sialyl-Lewis a on colon cancer cells promotes xenograft growth and angiogenesis in nude mice
}

\author{
Laura Terraneo ${ }^{\mathrm{a}}$, Laura Avagliano ${ }^{\mathrm{b}}$, Anna Caretti $^{\mathrm{a}}$, Paola Bianciardi ${ }^{\mathrm{a}}$, Delfina Tosi ${ }^{\mathrm{b}}$, \\ Gaetano Pietro Bulfamante ${ }^{\mathrm{b}}$, Michele Samaja ${ }^{\mathrm{a}}$, Marco Trincherac,* \\ a Department of Health Sciences, San Paolo Hospital, University of Milan, 20142 Milano, Italy \\ b Unit of Human Pathology, San Paolo Hospital, Department of Health Sciences, 20142 Milano, Italy \\ ${ }^{c}$ Department of Medicine Clinical and Experimental (DMCS), University of Insubria Medical School, 21100 Varese, Italy
}

\section{A R T I C L E I N F O}

\section{Article history:}

Received 2 May 2013

Received in revised form

23 September 2013

Accepted 1 October 2013

Available online 12 October 2013

\section{Keywords:}

Tumor marker

Colon cancer

Carbohydrate antigen

Tumor angiogenesis

E-selectin

\begin{abstract}
A B S T R A C T
We investigated the role of carbohydrate antigen sialyl-Lewis a (sLea), an E-selectin ligand and epitope of tumor marker CA19.9, in the development of xenografts in nude mice. To this end, animals were inoculated with the human colon cancer cell line HCT-15, expressing no Lewis antigens, or with a clone expressing sLea (HCT-15-T5). The size of HCT-15-T5 xenografts appeared larger than those of HCT-15 and their average weight was over twice bigger. In both xenografts the mitotic index was found elevated, as determined by Ki-67 assay, and no apoptosis was detected in the tumor cells by both caspase 8 or TUNEL assays. Some apoptotic signals were instead detected in the vessels. Conversely, microvessel density, determined through CD-31 immunohistochemistry, was found 3.2-folds bigger in HCT-15-T5 xenografts $(p<0.012)$. Only the membranes of HCT-15-T5 cells grown as xenografts reacted intensively with the anti CA19.9 antibody 1116-NS-19-9 by immunofluorescence, but not by immunohistochemistry. Unknown structures were instead stained by such technique in both xenografts, as were in mouse tissues not expressing the antigen and in human colon adenocarcinoma. We conclude that expression of sLea on the surface of colon cancer cells improves xenograft growth and is associated with enhanced angiogenesis, while immunohistochemistry with 1116-NS-19-9 antibody appears not suitable to determine CA19.9 expression.
\end{abstract}

(C) 2013 Elsevier Ltd. All rights reserved.

\section{Introduction}

Lewis antigens are a group of oligosaccharide residues constituting the end of various carbohydrate chains of glycoproteins and glycolipids protruding from the outer surface of mammalian cell membranes (Marionneau et al., 2001). Among them, sialylated Lewis antigens sLex and sLea were found to act as specific E-selectin ligands, and are thus potentially involved in many cell adhesion events (Läubli and Borsig, 2010). At present, sLex is a wellestablished E- and P-selectin ligand involved in leukocyte homing and neutrophil rolling to the vascular endothelium at the sites of inflammation (Sperandio, 2006). It was also found associated to malignancy when expressed on the surface of epithelial tumors,

Abbreviations: sLea, sialyl-Lewis a, NeuAc $\alpha 2-3$ Gal $\beta 1-3[$ Fuc $\alpha 1-4]$ GlcNAc; sLex sialyl-Lewis $x$, NeuAc $\alpha 2-3$ Gal $\beta 1-4[$ Fuc $\alpha 1-3]$ GlcNAc; H\&E, hematoxylin-eosin; TUNEL, terminal deoxynucleotidyl transferase dUTP nick end labeling.

* Corresponding author at: DMCS, via JH Dunant 5, 21100 Varese, Italy.

Tel.: +390332 397 160; fax: +390332397119.

E-mail address: marco.trinchera@uninsubria.it (M. Trinchera). and considered potentially involved in metastasis formation (St Hill, 2011).

sLea is an isomer of sLex, where the backbone disaccharide Gal $\beta 1,4 \mathrm{GlcNAc}$ (type 2 chain) is replaced by Gal $\beta 1,3 \mathrm{GlcNAc}$ (type 1 chain). The shift to type 1 chain synthesis is due to the expression of $\beta 1,3$ galactosyltransferase $\beta 3$ Gal-T5 (Isshiki et al., 1999). Due to the cell and tissue specific expression of $\beta 3 \mathrm{Gal}-\mathrm{T} 5$ versus the almost ubiquitous expression of $\beta 1$,4galactosyltransferases, type 1 chains are restricted to selected cell types. The biological role of sLea is unclear: it is present in the blood of many healthy individuals as the epitope of CA19.9 antigen, that is found elevated in various diseases, including cancers. At present it is recommended as a serum marker for the management of pancreatic cancer (Duffy et al., 2010). It was also proposed as an alternative to sLex in promoting cancer malignancy (Kannagi et al., 2004), although few experimental data are available so far. Moreover, the actual ability of colon cancer cells to synthesize sLea is controversial (Mare et al., 2013), since $\beta 3 \mathrm{Gal}-\mathrm{T5}$ is down-regulated in some cancers (Salvini et al., 2001; Isshiki et al., 2003; Caretti et al., 2012).

To address the function of sLea, we previously transfected the human colon cancer cell line HCT-15, which lacks expression of any Lewis antigen, with $\beta 3$ Gal-T5 cDNA and generate recombinant 
clones expressing type 1 chain Lewis antigens, mostly sLea, and no sLex (Mare and Trinchera, 2004). To assess whether or not sLea expression is maintained by tumor cells growing in vivo and affects tumor growth, a well characterized clone, named HCT-15-T5, together with the parental HCT-15 cells, was subcutaneously inoculated in nude mice, and the development of xenografts studied. In particular, we compared the growth and size of the xenografts, and characterized the main biochemical and morphological features.

\section{Materials and methods}

\subsection{Cells and transfected cell clone}

COLO-205 and SW-1116 cell lines were cultured as reported (Mare et al., 2013), rat cardiomyocytes H9c2 (ATCC CRL 1446) and mouse photoreceptor derived $661 \mathrm{~W}$ cells (a gift by Dr. R. Ghidoni, University of Milan) were cultured in DMEM containing 10\% fetal bovine serum, $100 \mathrm{U} / \mathrm{ml}$ penicillin, $1 \mathrm{mg} / \mathrm{ml}$ streptomycin, and $2 \mathrm{mM}$ L-glutamine. HCT-15-T5 clone was obtained by transfection of human colon cancer cell line HCT-15 (ATCC CCL-225) with the coding sequence of $\beta 3 \mathrm{Gal}-\mathrm{T} 5 \mathrm{cDNA}$ inserted in $\mathrm{pCDM} 8$ vector, as reported (Mare and Trinchera, 2004).

For microscopy studies, cells were harvested with 3 mM EDTA in PBS, washed in PBS, and centrifuged at room temperature for $5 \mathrm{~min}$ at $12,000 \times \mathrm{g}$. Pellets were soaked with $0.5 \mathrm{ml}$ of buffered $10 \%$ formalin solution, filtrated in filter bags, embedded in paraffin, and then processed for immunohistochemistry and immunofluorescence as for tissue sections.

\subsection{Xenografts}

Seven-week-old Foxn $1^{\text {nu/nu }}$ mice (Harlan), weighing 25-30 g at the entry into the study, were cared as reported (Terraneo et al., 2010). Cells were resuspended in PBS at a final concentration of $2 \times 10^{6} / 0.1 \mathrm{ml}$ and $0.1 \mathrm{ml}$ inoculated in each flank using a 26G insulin syringe starting from the same cell suspension for all the experiments. Xenograft growth was monitored as reported (Terraneo et al., 2010). After euthanasia by cervical dislocation, xenografts were quickly dissected away from surrounding skin and weighed. They were immediately cut in two parts: one frozen in liquid nitrogen and stored at $-80^{\circ} \mathrm{C}$ for biochemical analyses, and another fixed in formalin and embedded in paraffin for H\&E staining, immunohistochemistry, or immunofluorescence.

\subsection{DNA analysis}

Genomic DNA was extracted from xenograft specimens rid of capsular material, or from mouse liver or human HCT-15 cells, using a commercially available kit (QIAamp DNA, Qiagen) and submitted to competitive PCR as reported (Mare and Trinchera, 2007). Amplification program (30 cycles) included a single treatment at $94{ }^{\circ} \mathrm{C}$ for $3 \mathrm{~min}$ followed by cycles consisting of $1 \mathrm{~min}$ at $94^{\circ} \mathrm{C}$ (melting), $1.5 \mathrm{~min}$ at $66^{\circ} \mathrm{C}$ (annealing), $1.5 \mathrm{~min}$ at $72^{\circ} \mathrm{C}$ (extension), and a final extension step at $72{ }^{\circ} \mathrm{C}$ for $8 \mathrm{~min}$.

\subsection{Immunohistochemistry}

Immunohistochemical studies were carried out on $4 \mu \mathrm{m}$ thick tissue sections using a Novolink Polymer Detection System (Novocastra Laboratories) with the following primary antibodies. Ki-67 mouse monoclonal MIB1 (Dako), 1:100 dilution; CD-31 mouse monoclonal JC70A (Dako), 1:50 dilution; Caspase 8 rabbit monoclonal E6 (Abcam), 1:100 dilution; sLea mouse monoclonal 1116-NS-19-9 (ATCC HB-8059), 1:3000 dilution, prepared and purified as reported (Bardoni et al., 1999).
Sections were deparaffinized in BioClear (Bio Optica) for 20 min, washed twice in ethanol, and kept $30 \mathrm{~min}$ at $95^{\circ} \mathrm{C}$ in $9 \mathrm{mM}$ sodium citrate $\mathrm{pH}$ 6.0. Endogenous peroxidase activity was quenched with $3 \% \mathrm{H}_{2} \mathrm{O}_{2}$ for $10 \mathrm{~min}$. Staining was performed with 3,3diaminobenzidine as a chromogen. Slides were immunostained in the same batch, including negative controls lacking the primary antibody.

To quantify Ki-67 or caspase 8 immunostaining, the ratio between positive and total nuclei was calculated in five random microscopic areas at $60 \times$ by two independent observers with $100 \%$ agreement in the calculation of ratio. To quantify the number of intra-tumoral vessels, the number of $C D-31$ positive areas was calculated as for Ki- 67 but at $40 \times$. Necrotic areas were avoided during the calculation.

\subsection{Immunofluorescence}

Paraffin-embedded sections were deparaffinized with the following sequential treatments. Xylene, 3 times ( 5 min each); 100\% ethanol, twice ( 5 min each); $95 \%$ and $70 \%$ ethanol ( 3 min each); distilled water ( $30 \mathrm{~s})$, and PBS ( $5 \mathrm{~min})$. Sections were post-fixed with ethanol:acetic acid, $2: 1$ (vol:vol) at $-20^{\circ} \mathrm{C}$ for $5 \mathrm{~min}$, rinsed twice in PBS for $5 \mathrm{~min}$, boiled in $10 \mathrm{mM}$ citrate buffer, $\mathrm{pH} \mathrm{6.0,} \mathrm{for} 10 \mathrm{~min}$, cooled at room temperature for $20 \mathrm{~min}$, and washed twice in PBS.

Apoptosis was assessed using the TUNEL assay as already reported (Caretti et al., 2013). Total nuclei were counterstained with the karyophilic dye Hoechst 33258 (300 ng/ml, Sigma) for $3 \mathrm{~min}$ at room temperature, in the dark, followed by rinsing twice in PBS and cover slipping. Immunostaining with anti sLea (1:500) was performed according to the procedure of Terraneo et al. (2010).

\subsection{Statistical analysis}

To detect differences among the groups, we performed unpaired $t$ test with Welch correction.

\section{Results}

\subsection{Development of HCT-15 and HCT-15-T5 xenografts in nude mice}

The clone selected for the experiments (HCT-15-T5) was found to express $\beta 3 \mathrm{Gal}-\mathrm{T} 5$ activity and mRNA, as well as type 1 chain Lewis antigens, including sLea, as previously reported in details (Mare and Trinchera, 2004). sLea levels were similar to those of SW-1116 cells, a typical cell model for sLea studies. In a first experiment, nude mice receiving subcutaneous injection of HCT-15 and HCT-15-T5 were found to develop visible nodules at the sites of injection with high frequency. The size of HCT-15-T5 xenografts appeared larger than those of HCT-15. The difference was recognized after 2-3 weeks and became more evident in the following days (Fig. 1A, left panel). After 23 days, few xenografts appeared so big to start sacrificing the animals and collecting the masses. In other cases, they were allowed to growth for additional weeks. Such differences in growth rate are probably due to the intrinsic experimental variability. In the end, out of 8 animals injected on both sides with either cells, 14 xenografts were detected upon injection of both HCT-15 and HCT-15-T5 cells. The average weight of xenografts (Fig. 1A, right panel) was found to be 2.4-fold bigger in the case of HCT-15-T5, and such difference appears significant $(p<0.031)$. In a second experiment performed under identical conditions, all animals were sacrificed 30 days after the injection, and we obtained similar results (Fig. 1B). In fact, 16 and 15 xenografts were detected upon injection of HCT-15 and HCT-15-T5, respectively, and the size of those obtained with HCT-15-T5 was bigger: the average weight was twice larger than that with HCT-15 cells $(p<0.039)$. The collected tumor masses appeared rather pales in 


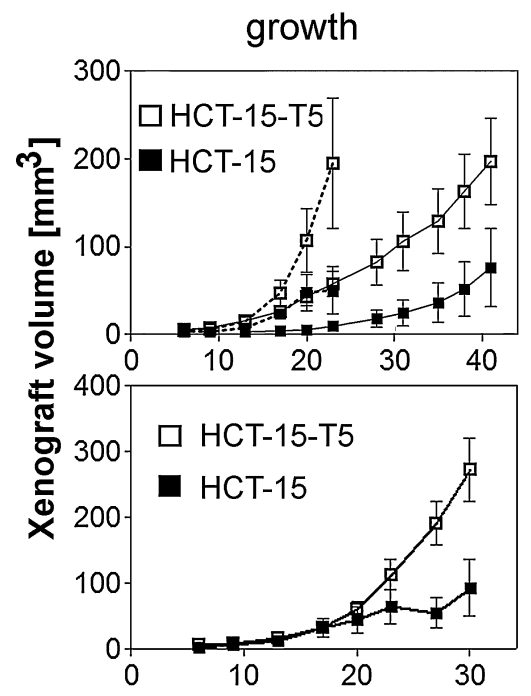

Time after xenograft [days]

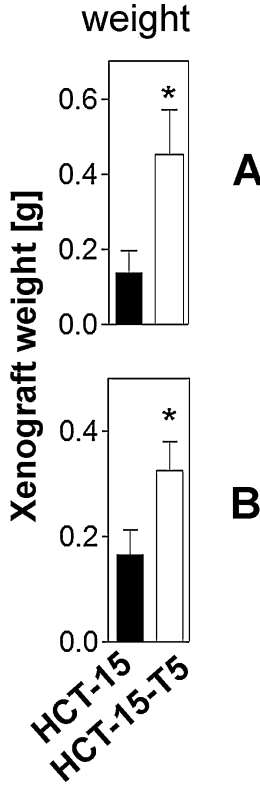

Fig. 1. Development of HCT-15 and HCT-15-T5 xenografts in nude mice. The growth of xenografts was determined three times a week as reported (Terraneo et al., 2010). (A) 3 out of 8 mice of each group (dotted lines) were sacrificed after 23 days and the xenografts removed, while the others were allowed to growth 2 additional weeks (full lines). Twelve xenografts were collected and weighted from each group. (B) All 16 animals were sacrificed after 30 days, and 9 or 14 xenografts were collected and weighted after HCT-15 or HCT-15-T5 injection, respectively. After euthanasia, xenografts were dissected away and weighed. The difference in the average xenograft weigh obtained from the 2 groups was found significant $(p<0.031$ and $p<0.039$ in experiments A and B, respectively).

both cases, and some xenografts contained amorphous fluid material released upon manipulation or cutting.

\subsection{Characterization of HCT-15 and HCT-15-T5 xenografts}

To understand the mechanisms underlying the differences in xenograft size, xenografts collected at 30 days post injection were analyzed by various techniques. Histologically, xenografts from both groups appear largely necrotic (Fig. 2A). In addition to cancer cells, an abundant stromal tree was evident, with some vessellike structures inside. DNA analysis showed that xenografts contain cells originated from the mouse host, as much as from the injected human cells. In fact, the amount of mouse and human genomic DNA detected in various xenograft specimens was similar, as determined by competitive PCR (supplemental Fig. S1), suggesting that only the epithelial cells derive from the injected human cancer cells, while the whole stroma was built around by the mouse host. The mitotic index was found elevated, as determined by Ki-67 assay, without differences between HCT-15 and HCT-15-T5 cells (Fig. 2B). No apoptosis was detected in both cases, by caspase 8 (Fig. 2C) or TUNEL (Fig. 2D) assays, in the tumor cells. However, some apoptotic signals were evident by both procedures in the vessels (see insets). These data suggest that the difference in size was not due to intrinsic differences in cell growth or cell survival, but instead to the interaction between the cells and the host tissue. We thus evaluated the presence and density of microvessels, determining the amount of CD-31 as a marker of vascular endothelium. Immunohistochemistry (Fig. 2E) showed that in the case of HCT-15 xenografts intratumoral CD-31 is rather poor, while in the case of HCT-15-T5 xenografts, that express sLea, it was 3.2-folds more evident $(p<0.012)$. Noteworthy, some vessel-like structures negative to $C D-31$ were recognized in both xenografts.

Supplementary material related to this article can be found, in the online version, at http://dx.doi.org/10.1016/ j.biocel.2013.10.003.
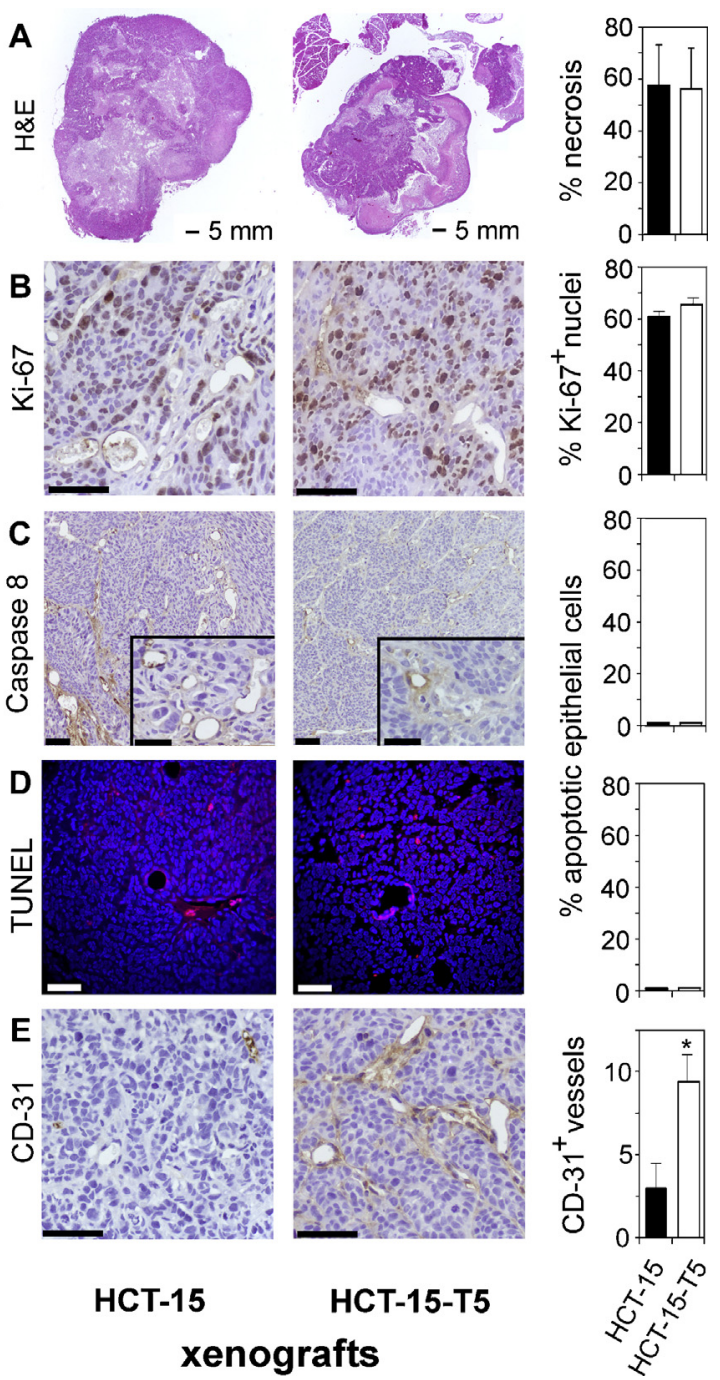

Fig. 2. Immunohistochemical characterization of xenografts. Xenografts developed in nude mice after inoculation of HCT-15 or HCT-15-T5 cells were processed as reported under Section 2. Results shown in the bar graphs are the mean \pm standard deviation for five random microscopic areas examined at the indicated magnification by two independent observers. Representative fields are presented. Scale bars are $100 \mu \mathrm{m}$ in the main fields (except H\&E) or $50 \mu \mathrm{m}$ in the insets. (A) H\&E staining of xenograft sections examined by light microscopy (magnification $2 \times$ ) showing conserved parenchyma (strong staining) and necrotic areas (pale zones). Percent of necrosis was calculated semi quantitatively by the ratio between pale and strong staining areas. (B) Ki-67 immunohistochemistry (magnification $60 \times$ ) showing abundant reactivity in both cases. (C) Caspase 8 immunohistochemistry (magnification $20 \times$ ) showing almost no apoptotic tumor cells in both xenografts. Inset: reactivity detected in some vessels (magnification 60x). (D) TUNEL assay of xenograft sections examined by fluorescence microscopy. Fragmented DNA ends were redlabeled by TMR while total nuclei were blue-counterstained with Hoechst 33258. Merged images are presented, where pink (apoptotic) nuclei are detected in putative endothelial cells of some vessels, but not in tumor cells of both xenografts (magnification $40 \times$ ). (E) CD-31 immunohistochemistry (magnification $60 \times$ ) showing more abundant reactivity in HCT-15-T5 xenografts. The number of reactive intra-tumoral vessels was calculated per microscopic field at $40 \times$ avoiding necrotic areas. The difference between xenografts was found significant $(p<0.012)$.

\subsection{Detection of sLea in the xenografts}

To confirm that parental HCT-15 cells do not express sLea when growing as a xenograft, while HCT-15-T5 do express the antigen, immunofluorescence with anti sLea was performed on the above sections using CA19.9 antibody (1116-NS-19-9). No fluorescence at all was detected in HCT-15 xenografts, while a bright positivity was found in HCT-15-T5 xenografts (Fig. 3, left side). Labeling was 


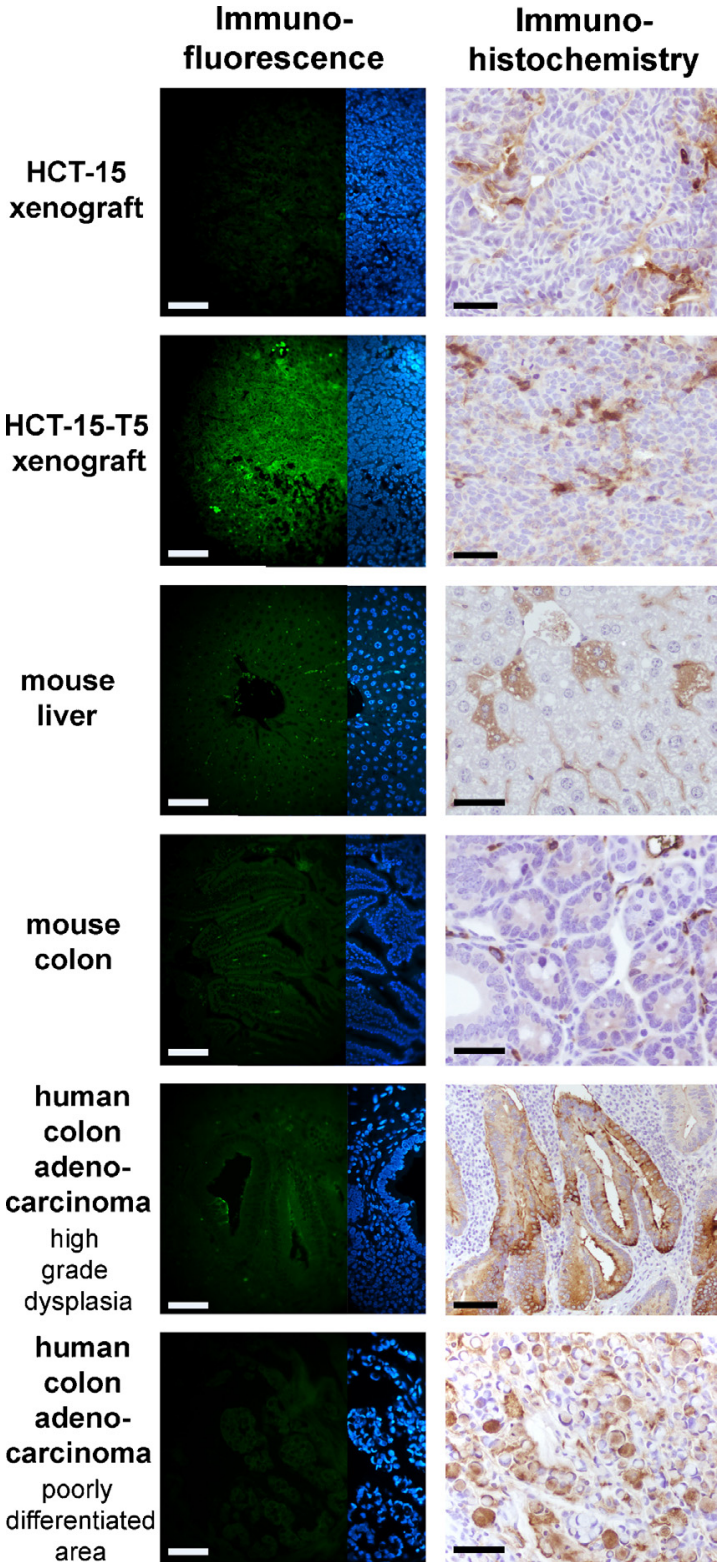

Fig. 3. Detection of sLea by immunofluorescence or immunohistochemistry. Sections were prepared and processed as reported under Section 2, following the procedure reported by Portela et al. (2011) for immunohistochemistry with 1116NS-19-9 antibody. Magnification is $40 \times$ for immunofluorescence, $40 \times$ (xenografts and high grade colon dysplasia) or $60 \times$ (mouse tissues and poorly differentiated area of colon adenocarcinoma) for immunohistochemistry. Scale bars $=100 \mu \mathrm{m}$ (immunohistochemistry of xenografts and high grade colon dysplasia) or $50 \mu \mathrm{m}$ (all others). Immunofluorescence detection of CA19.9 antigen is evident on the cell membranes of HCT-15-T5 xenograft only, which are not labeled by immunohistochemistry. Nuclei were blue-counterstained with Hoechst 33258 as reference (insets). Other unknown structures, not membrane-bound, are instead stained by immunohistochemistry in all sections. Note that immunohistochemistry results obtained with human colon cancer are very similar to those recently published (Portela et al., 2011).

typically located on the cell membrane, resembling the feature of HCT-15 cells growing in culture, and not in the stromal tree of mouse origin. Surprisingly, using the same antibody by immunohistochemistry, we found poor and opposite labeling of HCT-15-T5 xenografts (Fig. 3, right side). In fact, tumor cells were totally not reactive, while some reactivity was detected in the stroma, and not at the level of cell membranes. Even more surprisingly, the same pattern was also found in HCT-15 xenografts. We also stained tissue sections from different organs of the same mice as well as

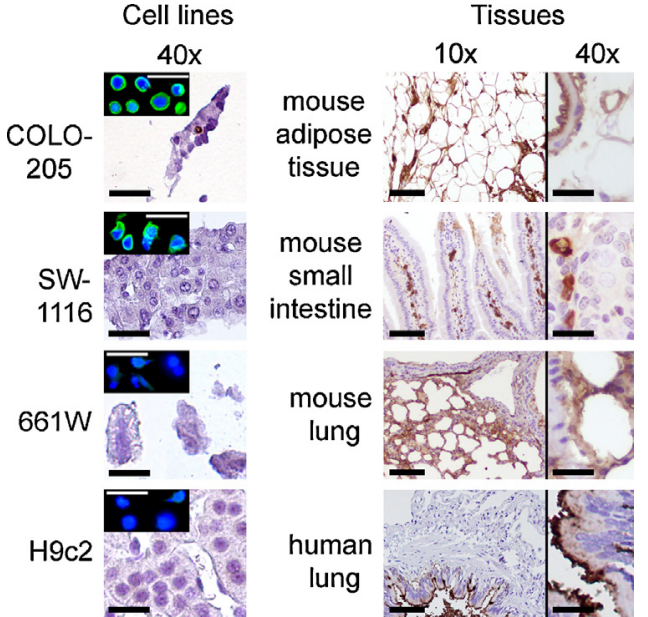

Fig. 4. Immunohistochemistry with anti sLea antibody 1116-NS-19-19 in reference cell lines. Cultured cell lines were harvested with $3 \mathrm{mM}$ EDTA, washed in PBS, filtrated in filter bags, embedded in paraffin, and then processed for immunohistochemistry and immunofluorescence as for tissue sections in Fig. 3. Scale bars $=100 \mu \mathrm{m}$. Insets: merged images are presented for immunofluorescence, where blue nuclei are detected in all cells and enclosed into green plasma membranes in positive cells only. No reactive cells were detected by immunohistochemistry. For comparison, other tissues reactive under the reported conditions are shown on the right side. Scale bars $=400 \mu \mathrm{m}$ (magnification $10 \times$ ), or $100 \mu \mathrm{m}$ (magnification $40 \times$ ). Note the reactivity of adipocytes and vessels in the mouse adipose tissue, the lack of reactivity of enterocytes in the mouse small intestine, where instead some blood-borne cells (presumably macrophages) are strongly reactive, and the diffuse reactivity of pneumocytes in the mouse lung versus the restricted reactivity of the bronchiolar surface in the human lung.

from an independent C57BL/6 mouse. We did find reactivity in various sections of all mice, including the colon and the liver (Fig. 3) Interestingly, we found a similar pattern by CA.19.9 immunohistochemistry of a human colon adenocarcinoma (Fig. 3), that instead was almost negative by immunofluorescence, as were all the mouse sections.

To confirm the result (Fig. 4), we stained two reference cell lines as positive controls, COLO-205 and SW-1116. The former expresses the highest levels of sLea reported (Mare and Trinchera, 2004), and the latter is the one originally used as immunogen for developing 1116-NS-19-9 hybrydoma (Koprowski et al., 1979). Rodent cell lines were stained as the negative controls. Utilizing the identical procedure used for the above tissue sections, even COLO-205 and SW-1116 were totally negative by innunohistochemistry, while other tissues, as the lung, the small intestine, and the adipose tissue, were clearly reactive. This indicates that the antibody detects no sLea at all by immunohistochemistry, but instead unknown molecules commonly expressed in mouse and human tissues.

\section{Discussion}

In this paper we found that the expression of sLea on the surface of colon cancer cells HCT-15 improves xenograft growth and is associated with enhanced angiogenesis.

HCT-15 cells appeared highly tumorigenic in nude mice; in fact, we obtained successful xenografts after almost all injections. Such cells replicated very fast and escaped apoptosis, as assessed by Ki-67, caspase 8 , and TUNEL assays, but failed to give raise to very large tumors and quickly became necrotic. We hypothesize that HCT-15 cells grow faster than the rate they stimulate vascularization, probably because they lack to express multiple angiogenic stimuli. In fact, few CD-31 reactive microvessels were recognizable inside the xenograft structure. On the other side the presence of the E-selectin ligand sLea on the surface of HCT-15-T5 xenografts was associated with a recognizable network of CD-31 
positive microvessels that appeared able, at least in part, to sustain cell growth. This suggests that sLea is able to restore the angiogenic stimulus, at least in part, probably acting as a ligand for E-selectin expressed by endothelial cells. We also found that xenografts derived from both HCT-15 and HCT-15-T5 cells showed an abundant stromal architecture derived from the host where some endothelial-like cells were present, but appeared CD-31 negative. Since we found that some of them were apoptotic, we speculate that such $C D-31$ negative endothelial-like cells may be not living, and not able to give rise to functional vessels. Conversely, in the presence of sLea, at least a part of the recruited endothelial cells may remain alive and maintain functional vessels. This would be in agreement with the role already assigned to other adhesion molecules in specific steps of angiogenesis (Ramjaun and Hodivala-Dilke, 2009) and with that specifically suggested for E-selectin in melanoma growth (Liu et al., 2011). Interestingly, in another model of xenograft growth, the selectin ligand isomer sLex provided similar angiogenic effects (Mathieu et al., 2007). Previously, an angiogenic role of sLex and sLea together was reported in a study that involved the cotransplantation of cancer and endothelial cells (Tei et al., 2002). To our knowledge, data here presented are the first demonstrating the actual ability of sLea alone to promote tumor angiogenesis in a nude mice model.

Another relevant finding of the present study is the evidence that the anti-sLea antibody 1116-NS-19-9 is not suitable for immunohistochemistry. In fact, we found that HCT-15-T5 xenografts, where the membranes of epithelial cells were well reactive with the antibody by immunofluorescence, were not by immunohistochemistry. Even COLO-205 and SW-1116 cells, which are the prototype of cells expressing sLea, were not stained by immunohistochemistry. Moreover, various unknown structures that were negative by immunofluorescence did react by immunohistochemistry even in HCT-15 xenografts, as well as in various mouse tissues. Since sLea is not synthesized in the rodents (Falk et al., 1995), due to the lack of any $\alpha 1,4$ fucosyltransferase activity (Dupuy et al., 2002), it is evident that 1116-NS-19-9 antibody does not recognized sLea by immunohistochemistry but instead other structures unknown at present, not located on the cell membrane. Interestingly, a similar reactivity pattern was also found in a colon adenocarcinoma. Apparently, normal colon mucosa cells do not synthesize such unknown molecules, which become detectable during neoplastic transformation being produced by the tumor itself or by the surrounding tissue. Since histochemical reactivity with such antibody is sometimes assumed as a detection of CA19.9 antigen in cancer tissues (Portela et al., 2011), such data should be cautiously re-evaluated. This is particularly relevant in colon adenocarcinomas, since we recently found that their glycosylation machinery is unable to sustain relevant synthesis of sLea (Mare et al., 2013), opening a question about the origin of the CA19.9 antigen circulating in colon cancer patients. Taken together, our recent and present findings indicate that neither the value of serum CA19.9 nor the immunohistochemical detection of CA19.9 by $1116-$ NS-19-9 antibody in cancer sections predict the actual expression of the antigen by colon cancer cells, as it has been assumed so far. On the other side, they suggest that the assessment of the actual sLea status of a colon cancer may be very important for the prognosis, since we proved that expression of the true antigen promotes angiogenesis and, in turn, tumor growth.

\section{Acknowledgments}

This work was supported by grants from Mizutani Foundation for Glycosciences and from the University of Insubria to MT.

\section{References}

Bardoni A, Valli M, Trinchera M. Differential expression of beta1,3galactosyltransferases in human colon cells derived from adenocarcinomas or normal mucosa. FEBS Lett 1999;451:75-80.

Caretti A, Sirchia SM, Tabano S, Zulueta A, Dall'Olio F, Trinchera M. DNA methylation and histone modifications modulate the $\beta 1,3$ galactosyltransferase $\beta 3 \mathrm{Gal}-\mathrm{T5}$ native promoter in cancer cells. Int J Biochem Cell Biol 2012:44:84-90.

Caretti A, Bianciardi P, Marini M, Abruzzo PM, Bolotta A, Terruzzi C, et al. Supplementation of creatine and ribose prevents apoptosis and right ventricle hypertrophy in hypoxic hearts. Curr Pharm Des 2013(April) [Epub ahead of print] PMID 23590158.

Duffy MJ, Sturgeon C, Lamerz R, Haglund C, Holubec VL, Klapdor R, et al. Tumor markers in pancreatic cancer: a European Group on Tumor Markers (EGTM) status report. Ann Oncol 2010;21:441-7.

Dupuy F, Germot A, Marenda M, Oriol R, Blancher A, Julien R, et al. Alpha1,4-fucosyltransferase activity: a significant function in the primate lineage has appeared twice independently. Mol Biol Evol 2002;19:815-24.

Falk PG, Bry L, Holgersson J, Gordon JI. Expression of a human alpha-1,3/4-fucosyltransferase in the pit cell lineage of $\mathrm{FVB} / \mathrm{N}$ mouse stomach results in production of Leb-containing glycoconjugates: a potential transgenic mouse model for studying Helicobacter pylori infection. Proc Nat Acad Sci U S A 1995;92:1515-9.

Gilliland G, Perrin S, Blanchard K, Bunn HF. Analysis of cytokine mRNA and DNA: detection and quantitation by competitive polymerase chain reaction. Proc Natl Acad Sci U S A 1990;87:2725-9.

Isshiki S, Kudo T, Nishihara S, Ikehara Y, Togayachi A, Furuya A, et al. Lewis type 1 antigen synthase (beta3Gal-T5) is transcriptionally regulated by homeoproteins. J Biol Chem 2003;278:36611-20.

Isshiki S, Togayachi A, Kudo T, Nishihara S, Watanabe M, Kubota $\mathrm{T}$, et al. Cloning, expression, and characterization of a novel UDP-galactose: $\beta$-N-acetylglucosamine $\beta 1,3$-galactosyltransferase $(\beta 3 \mathrm{Gal}-\mathrm{T} 5)$ responsible for synthesis of type 1 chain in colorectal and pancreatic epithelia and tumor cells derived therefrom. J Biol Chem 1999;274:12499-507.

Kannagi R, Izawa M, Koike T, Miyazaki K, Kimura N. Carbohydrate-mediated cell adhesion in cancer metastasis and angiogenesis. Cancer Sci 2004:95:377-84.

Koprowski H, Steplewski Z, Mitchell K, Herlyn M, Herlyn D, Fuhrer P. Colorectal carcinoma antigens detected by hybridoma antibodies. Somatic Cell Genet 1979;5:957-71.

Läubli H, Borsig L. Selectins promote tumor metastasis. Semin Cancer Biol 2010;20:169-77.

Liu ZJ, Tian R, Li Y, An W, Zhuge Y, Livingstone AS, et al. Inhibition of tumor angiogenesis and melanoma growth by targeting vascular E-selectin. Ann Surg 2011;254:450-7.

Mare L, Caretti A, Albertini R, Trinchera M. CA19.9 antigen circulating in the serum of colon cancer patients: where is it from? Int J Biochem Cell Biol 2013:45:792-7.

Mare L, Trinchera M. Comparative analysis of retroviral and native promoters driving expression of beta1,3-galactosyltransferase beta3Gal-T5 in human and mouse tissues. J Biol Chem 2007;282:49-57.

Mare L, Trinchera M. Suppression of $\beta 1$,3galactosyltransferase $\beta 3$ Gal-T5 in cancer cells reduces sialyl-Lewis a and enhances poly $\mathrm{N}$-acetyllactosamines and sialyl-Lewis x on O-glycans. Eur J Biochem 2004;271:186-94.

Marionneau S, Cailleau-Thomas A, Rocher J, Le Moullac-Vaidye B, Ruvoën N Clément $\mathrm{M}$, et al. ABH Lewis histo-blood group antigens, a model for the meaning of oligosaccharide diversity in the face of a changing world. Biochimie 2001;83:565-73.

Mathieu S, Gerolami R, Luis J, Carmona S, Kol O, Crescence L, et al. Introducing alpha(1,2)-linked fucose into hepatocarcinoma cells inhibits vasculogenesis and tumor growth. Int J Cancer 2007:121:1680-9.

Portela SV, Martín CV, Romay LM, Cuevas E, Martín EG, Briera AF. sLea and sLex expression in colorectal cancer: implications for tumourigenesis and disease prognosis. Histol Histopathol 2011;26:1305-16.

Ramjaun AR, Hodivala-Dilke K. The role of cell adhesion pathways in angiogenesis. Int J Biochem Cell Biol 2009;41:521-30.

Salvini R, Bardoni A, Valli M, Trinchera M. Beta 1,3-Galactosyltransferase beta 3 Gal-T5 acts on the GlcNAcbeta $1 \rightarrow 3$ Galbeta $1 \rightarrow 4$ GlcNAcbeta $1 \rightarrow R$ sugar chains of carcinoembryonic antigen and other N-linked glycoproteins and is down-regulated in colon adenocarcinomas. J Biol Chem 2001;276: 3564-73.

Sperandio M. Selectins and glycosyltransferases in leukocyte rolling in vivo. FEBS J 2006;273:4377-89.

St Hill CA. Interactions between endothelial selectins and cancer cells regulate metastasis. Front Biosci 2011;16:3233-51.

Tei K, Kawakami-Kimura N, Taguchi O, Kumamoto K, Higashiyama S, Taniguchi $\mathrm{N}$, et al. Roles of cell adhesion molecules in tumor angiogenesis induced by cotransplantation of cancer and endothelial cells to nude rats. Cancer Res 2002;62:6289-96.

Terraneo L, Bianciardi P, Caretti A, Ronchi R, Samaja M. Chronic systemic hypoxia promotes LNCaP prostate cancer growth in vivo. Prostate 2010;70: 1243-54. 